\title{
2q24 microdeletion syndrome
}

INSERM

\section{Source}

INSERM. (1999). Orphanet: an online rare disease and orphan drug data base. 2 q24 microdeletion syndrome. ORPHA:1617

2q24 microdeletion syndrome is a chromosomal anomaly consisting of a partial long arm deletion of chromosome 2 and characterized clinically by a wide range of manifestations (depending on the specific region deleted) which can include seizures, microcephaly, dysmorphic features, cleft palate, eye abnormalities (coloboma, cataract and microphthalmia), growth retardation, failure to thrive, heart defects, limb anomalies, developmental delay and autism. 